\title{
Targeted SMN Exon Skipping: A Useful Control to Assess In Vitro and In Vivo Splice-Switching Studies
}

\author{
Loren L. Flynn ${ }^{1,2,3,4}{ }^{\oplus}$, Chalermchai Mitrpant ${ }^{2,5}{ }^{\oplus}$, Abbie Adams ${ }^{1,2,3}{ }^{\oplus}$, Ianthe L. Pitout ${ }^{1,6}$, Anja Stirnweiss ${ }^{6}$, \\ Sue Fletcher $1,3,6$ and Steve D. Wilton ${ }^{1,2,3, *}$ \\ 1 Centre for Molecular Medicine and Innovative Therapeutics, Health Futures Institute, Murdoch University, \\ Murdoch, WA 6150, Australia; loren.flynn@murdoch.edu.au (L.L.F.); a.adams@murdoch.edu.au (A.A.); \\ i.pitout@murdoch.edu.au (I.L.P.); S.fletcher@murdoch.edu.au (S.F.) \\ 2 Perron Institute for Neurological and Translational Science, Nedlands, WA 6009, Australia; \\ chalermchai.mit@mahidol.edu \\ 3 Centre for Neuromuscular \& Neurological Disorders, University of Western Australia, \\ Crawley, WA 6009, Australia \\ 4 Black Swan Pharmaceuticals, Wake Forest, NC 27587, USA \\ 5 Department of Biochemistry, Faculty of Medicine Siriraj Hospital, Mahidol University, \\ Bangkok 10700, Thailand \\ 6 PYC Therapeutics, Nedlands, WA 6009, Australia; anja.stirnweiss@pyctx.com \\ * Correspondence: s.wilton@murdoch.edu.au
}

Citation: Flynn, L.L.; Mitrpant, C.; Adams, A.; Pitout, I.L.; Stirnweiss, A.; Fletcher, S.; Wilton, S.D. Targeted SMN Exon Skipping: A Useful Control to Assess In Vitro and In Vivo Splice-Switching Studies. Biomedicines 2021, 9, 552. https://doi.org/ 10.3390/biomedicines 9050552

Academic Editor: Bernard Lebleu

Received: 16 April 2021

Accepted: 11 May 2021

Published: 14 May 2021

Publisher's Note: MDPI stays neutral with regard to jurisdictional claims in published maps and institutional affiliations.

Copyright: (c) 2021 by the authors. Licensee MDPI, Basel, Switzerland. This article is an open access article distributed under the terms and conditions of the Creative Commons Attribution (CC BY) license (https:// creativecommons.org/licenses/by/ $4.0 /)$.

\begin{abstract}
The literature surrounding the use of antisense oligonucleotides continues to grow, with new disease and mechanistic applications constantly evolving. Furthermore, the discovery and advancement of novel chemistries continues to improve antisense delivery, stability and effectiveness. For each new application, a rational sequence design is recommended for each oligomer, as is chemistry and delivery optimization. To confirm oligomer delivery and antisense activity, a positive control AO sequence with well characterized target-specific effects is recommended. Here, we describe splice-switching antisense oligomer sequences targeting the ubiquitously expressed human and mouse SMN and Smn genes for use as control AOs for this purpose. We report two AO sequences that induce targeted skipping of SMN1/SMN2 exon 7 and two sequences targeting the Smn gene, that induce skipping of exon 5 and exon 7 . These antisense sequences proved effective in inducing alternative splicing in both in vitro and in vivo models and are therefore broadly applicable as controls. Not surprisingly, we discovered a number of differences in efficiency of exon removal between the two species, further highlighting the differences in splice regulation between species.
\end{abstract}

Keywords: antisense oligonucleotide; morpholino; positive control; survival motor neuron; cell penetrating peptide

\section{Introduction}

Antisense oligonucleotides (AOs) are synthetic nucleic acid analogues, specifically designed to regulate target gene transcripts and protein expression. Common mechanistic strategies include: RNase H-mediated transcript degradation [1]; preventing the initiation of protein translation [2]; and modulating pre-mRNA splicing to either produce alternative functional protein isoforms, to overcome mutations or errors in splicing, or to produce a non-functional transcript [3-6]. The application of splice-switching antisense therapies to treat a broad variety of genetic disorders has greatly expanded over the past decade, with antisense drugs to treat Duchenne muscular dystrophy and spinal muscular atrophy now available in the clinic. For a review of the translational development of splice-switching antisense therapies, see Pitout et al, 2019 [7].

We have previously reported our recommendations for the efficient and rational design of $\mathrm{AO}$ sequences for splice-modulation, as well as the optimization of delivery and choice of $\mathrm{AO}$ chemistry for improved splice-switching effects [8,9]. In particular, 
AO delivery is of paramount importance and hence should be optimized through the comparison of multiple transfection reagents and delivery techniques when a new gene transcript or cell line is investigated. Traditionally, the use of a fluorescently tagged $\mathrm{AO}$ has been recommended for optimization of transfection efficiency [10,11]; however, the addition of a fluorescent tag or similar conjugate increases the molecular weight of the $\mathrm{AO}$ and likely influences the uptake of the compound, while cleavage of the tag within the cytoplasm may confound interpretation of AO localization. Visualization of a fluorescently labelled $\mathrm{AO}$ is limited in identifying that the $\mathrm{AO}$ permeates the cell but does not confirm $\mathrm{AO}$ escape from the endosome, which is necessary for biological availability and activity of the AO [12,13]. Furthermore, fluorescent AOs are unsuitable for in vivo applications. An antisense sequence targeting a ubiquitously expressed and efficiently modified transcript is, therefore, an ideal compound for transfection optimization and for use as a positive control in subsequent splice manipulation experiments. The effects of such a splice-switching control AO can allow quantitation of transfection efficiency and can characterize optimal transfection parameters. Importantly, the use of a positive control $\mathrm{AO}$ targeting a ubiquitously expressed gene serves as an ideal positive control for broad tissue targeting and in vivo evaluation of new $\mathrm{AO}$ chemistries and modifications.

We have extensive experience with developing $\mathrm{AO}$ sequences for exon skipping and routinely screen 2'-O-methyl phosphorothioate (PS) AOs and phosphorodiamidate morpholinos (PMOs) to identify lead drug candidates. While 2'-O-methyl PS AOs are suitable for in vitro screening to identify the optimal target binding site, off-target binding and adverse events due to the negatively charged phosphorothioate backbone make them less suitable for in vivo and clinical applications [14-16]. Conversely, PMOs have an excellent safety profile, and when efficiently delivered, are more effective than their 2'-O-methyl counterparts when studying protein changes or functional assays [17]. Delivery of PMOs in vivo typically requires high dosing and/or frequent administration [18]. Therefore, efforts to enhance delivery have focused on conjugating PMOs to cell penetrating peptides (PPMOs), improving cellular uptake by up to 10-fold [18-21]. We have developed a robust positive control AO targeting the $S M N / S m n$ transcripts that can be used to optimize and evaluate delivery of antisense nucleic acid analogues and identify tissue-specific or differential delivery in vivo.

The survival motor neuron (SMN) protein is ubiquitously expressed, and its reduced expression is associated with the childhood disease spinal muscular atrophy (SMA) due to the functional loss of the SMN1 gene. A single base change in exon 7 between the two genes encoding SMN, SMN1 and SMN2 modifies splicing factor binding, leading to the alternative splicing of that in-frame exon (Figure 1a). While AOs targeting an intronic silencer element downstream of SMN2 exon 7 have been developed to increase SMN levels to overcome SMA [22-24], AOs inducing skipping of exon 7 from the SMN1 transcript can be used to induce and model disease [25], as well as act as positive controls for separate AO studies. Here, we report sequences targeting in-frame exons 5 and 7 of the SMN1/SMN2 and Smn transcripts that can be used as antisense controls for optimizing AO delivery and chemistry in both in vitro and in vivo models. Not surprisingly, we again observed substantial variation in the efficiencies of exons 5 and 7 removal, between human and mouse transcripts, highlighting differences in splice-regulation between species [26]. 
(a) SMN1 Exon 7

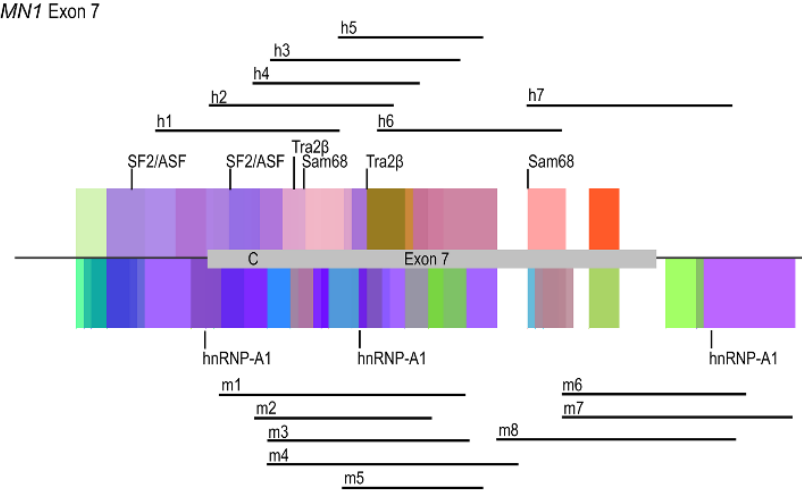

SMN2 Exon 7

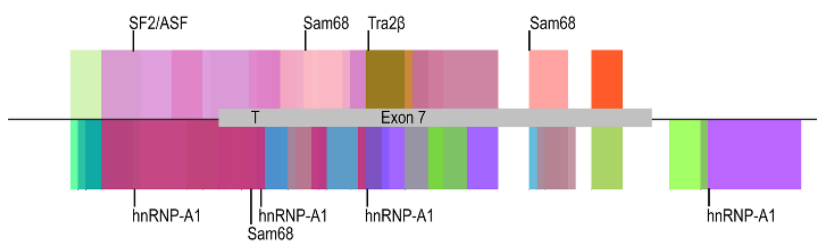

(b) $S M N 1 / 2$ Exon 5

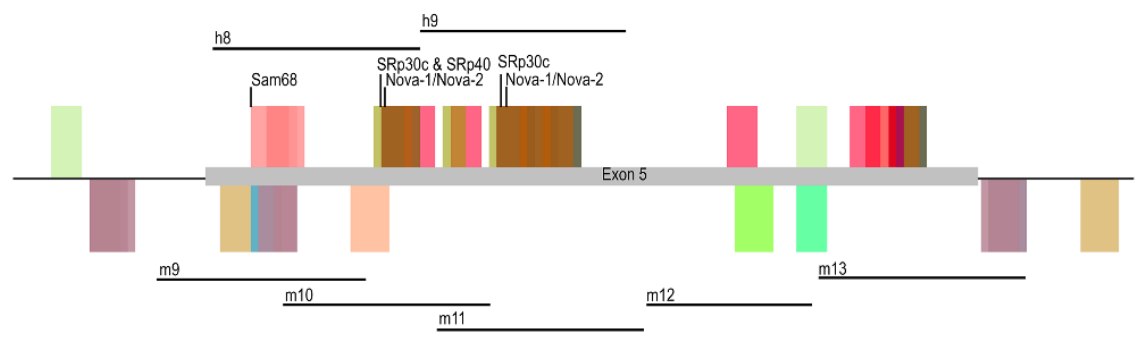

(c)
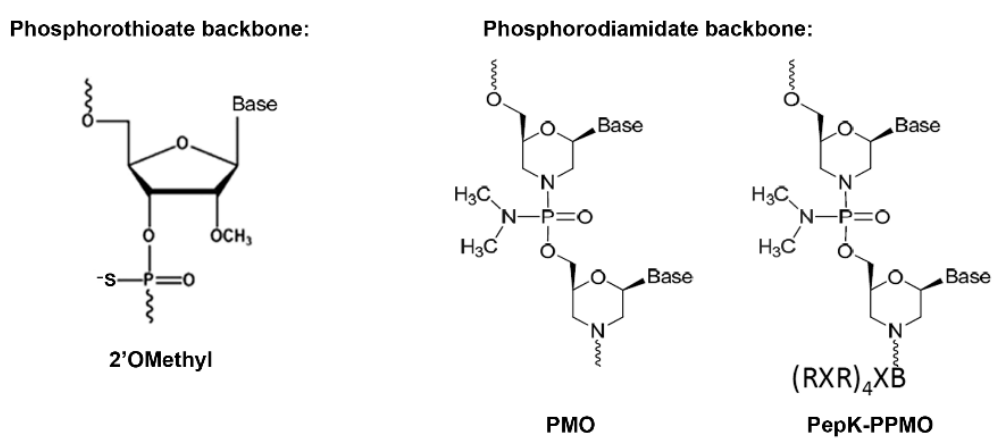

Figure 1. (a) schematic of SMN1 and SMN2 exon 7 SpliceAid predicted splicing factor binding sites with the more strongly predicted splicing factors labelled, including alternative splicing factor/pre-mRNA splicing factor 2 (ASF/SF2) and transformer 2 Beta (Tra2 $\beta$ ) as positive exonic splicing enhancer elements, and SRC associated in mitosis of $68 \mathrm{kDa}$ (Sam68) and heterogenous nuclear ribonucleoprotein A1 (hnRNP-A1) as negative exonic splicing silencer elements. The binding location of the human exon 7 skipping AOs are indicated above the SMN1 exon 7, and the homologous mouse Smn exon 7 skipping AOs are indicated below; (b) schematic of SMN1/2 exon 5 predicted splicing factor binding sites, with the more strongly predicted splicing factors labelled, including Sam68, serine and arginine rich splicing factors (SRp-30c and SRp-40) and the Nova-1 and Nova-2 proteins as exonic splicing enhancers. The human and mouse exon 5 skipping AOs are indicated above and below the schematic, respectively; and (c) the chemical structure of the three AO chemistries used in this study, including the 2'-O-methyl phosphorothioate AO, the PMO and the PepK-conjugated PPMO, adapted from $[27,28]$.

\section{Materials and Methods}

\subsection{AO Design}

AOs were initially designed to induce skipping of exon 7 from the human and mouse $S M N / S m n$ genes, and following these results, to induce exon 5 skipping in both species. Human-specific AOs were designed to target exonic splicing enhancer (ESE) domains as predicted by the online SpliceAid prediction tool, available at http:/ / www.introni.it/ splicing.html, last accessed on 9 May 2021 [29]. Mouse-specific AOs were designed to target ESEs as predicted by the online Rescue-ESE program, available at http://genes. mit.edu/burgelab/rescue-ese/, last accessed on 19 December 2014 [30]. The predicted 
splicing factor binding sites and the AO target sites for both the human and mouse exons are illustrated in Figure 1a,b respectively. AO nomenclature was based on that described by Mann et al. (2002) [31]. All 2'-O-methyl AOs were synthesized in house on an Expedite 8909 nucleic acid synthesizer with a phosphorothioate (PS) backbone. All AOs tested in this study, their sequences and binding coordinates are listed in Table 1 . The chemical structure for each of the AO chemistries utilized in this study, the $2^{\prime}-O$-methyl phosphorothioate, the phosphorodiamidate morpholino (PMO) and the PepK-conjugated PMO are illustrated in Figure 1c.

Table 1. Sequences and binding coordinates of exon skipping AOs designed for the human SMN and mouse Smn transcripts. The AO length and melting temperature (Tm) of the 2'-O-methyl AO sequence is listed.

\begin{tabular}{|c|c|c|c|c|c|}
\hline $\begin{array}{l}\text { Species and } \\
\text { Exon }\end{array}$ & $\begin{array}{c}\text { AO } \\
\text { Name/Number }\end{array}$ & Coordinates & Sequence $5^{\prime}-3^{\prime}$ & $\begin{array}{l}\text { Length } \\
\text { (bp) }\end{array}$ & $\operatorname{Tm}\left({ }^{\circ} \mathrm{C}\right)$ \\
\hline \multirow{7}{*}{$\begin{array}{l}\text { Human } \\
\text { Exon } 7\end{array}$} & h1 & SMN H7A $(-08+17)$ & UGA UUU UGU CUA AAA CCC UGU AAG G & 25 & 66.4 \\
\hline & h2 & SMN H7A $(+01+25)^{1}$ & CUU CUU UUU GAU UUU GUC UAA AAC C & 25 & 58.6 \\
\hline & h3 & SMN H7A $(+07+31)^{1}$ & ACC UUC CUU CUU UUU GAU UUU GUC U & 25 & 64.7 \\
\hline & h4 & SMN H7A $(+06+27)^{1}$ & UCC UUC UUU UUG AUU UUG UCU G & 22 & 59.5 \\
\hline & h5 & $\operatorname{SMN~H7A}(+13+32)^{1}$ & CAC CUU CCU UCU UUU UGA UU & 20 & 59.2 \\
\hline & h6 & SMN H7A $(+22+46)^{1}$ & UUA AGG AAU GUG AGC ACC UUC CUU C & 25 & 70.5 \\
\hline & h7 & SMN H7D $(+17-13)$ & CUG GCA GAC UUA CUC CUU AAU UUA AGG AAU & 30 & 70.2 \\
\hline Human & h8 & SMN H5A(+ $04+28)$ & GUG GUG GGC CAU UGA AUU UUA GAC C & 25 & 71.1 \\
\hline Exon 5 & h9 & SMN H5A $(+29+53)$ & UGG GGU GGU GGU GGU GGC GGU GGC G & 25 & 87.0 \\
\hline \multirow{8}{*}{$\begin{array}{l}\text { Mouse } \\
\text { Exon } 7\end{array}$} & m1 & Smn M7A $(+02+31)$ & ACU UUC CUU CUU UUU UAU UUU GUC UGA AAC & 30 & 63.5 \\
\hline & $\mathrm{m} 2$ & Smn M7A $(+06+27)$ & UCC UUC UUU UUU AUU UUG UCU G & 22 & 56.9 \\
\hline & $\mathrm{m} 3$ & Smn M7A $(+07+31)$ & ACU UUC CUU CUU UUU UAU UUU GUC U & 25 & 59.8 \\
\hline & $\mathrm{m} 4$ & Smn M7A $(+07+36)^{2}$ & UGA GCA CUU UCC UUC UUU UUU AUU UUG UCU & 30 & 67.2 \\
\hline & m5 & Smn M7A $(+13+32)$ & CAC UUU CCU UCU UUU UUA UU & 20 & 52.6 \\
\hline & m6 & Smn M7D $(+11-14)$ & AAU GAC AGA CUU ACU UCU UAA UUU G & 25 & 60.7 \\
\hline & $\mathrm{m} 7$ & Smn M7D(+ $11-19)$ & UUU AAA AUG ACA GAC UUA CUU CUU AAU UUG & 30 & 61.9 \\
\hline & $\mathrm{m} 8$ & Smn M7D(+ $17-13)$ & AUG ACA GAC UUA CUU CUU AAU UUG UAU GUG & 30 & 65.9 \\
\hline \multirow{5}{*}{$\begin{array}{l}\text { Mouse } \\
\text { Exon } 5\end{array}$} & $\mathrm{~m} 9$ & Smn M5A $(-06+19)$ & CGU UGA AUU UUA GAC CUG GCU AUA A & 25 & 65.8 \\
\hline & $\mathrm{m} 10$ & Smn M5A $(+12+36)$ & AGG CGG CGG CGG CGG GCC GUU GAA U & 25 & 86.8 \\
\hline & $\mathrm{m} 11$ & Smn M5A $(+32+54)$ & AAG GGG GGA GGG GGU AGU GGA GGC G & 25 & 85.9 \\
\hline & $\mathrm{m} 12$ & Smn M5A $(+56+77)$ & AAC GGG GGC AUC CAG CAC GGC AG & 23 & 81.6 \\
\hline & $\mathrm{m} 13$ & Smn M5A(+ $18-07)$ & UAC UUA CUG GUG GUC CUG AAG GGA A & 25 & 75.7 \\
\hline
\end{tabular}

${ }^{1}$ Some sequence overlap with $2^{\prime}$-O-methoxyethyl AOs described in [25]. ${ }^{2}$ Previously used as a control [4].

\subsection{Cell Plating and 2'-O-Methyl Phosphorothioate AO Transfection}

Normal human dermal fibroblasts prepared in-house (Murdoch University Human Research Ethics Committee Approval \#2013/156) were proliferated and seeded $\left(7500 \mathrm{cells} / \mathrm{cm}^{2}\right)$ in $10 \%$ FBS DMEM and incubated overnight prior to $2^{\prime}-O$-methyl PS-AO transfection. Transfections were performed using Lipofectamine 3000 (Life Technologies, Melbourne, Australia) with $3 \mu \mathrm{L}$ of Lipofectamine 3000 per $1 \mathrm{~mL}$ of transfection volume, according to the manufacturer's protocol, and incubated for $48 \mathrm{~h}$. AO-lipid complexes were made up in OptiMEM (Life Technologies).

Primary mouse $m d x$ myoblasts were seeded onto Matrigel (Becton Dickinson Life Technologies) pre-coated plates $\left(100 \mu \mathrm{g} / \mathrm{mL}, 37^{\circ} \mathrm{C}\right.$ for $\left.1 \mathrm{~h}\right)$ in $20 \%$ FBS, $0.5 \%$ chick embryo extract, Ham's-F10 and incubated overnight prior to transfection. 2'-O-methyl PS-AOs were transfected into mouse cells using Lipofectin (Life Technologies, Melbourne, Australia) at a 2:1 ratio of Lipofectin to total AO, in OptiMEM, according to manufacturer's protocols, and incubated for $48 \mathrm{~h}$.

\subsection{Culture and Differentiation of SH-SY5Y Cells}

The SH-SY5Y neuroblastoma cell line was maintained in 10\% FBS EMEM/HamsF12 (1:1). For differentiation into neuronal-like cells, SH-SHY5Y cells were plated onto Matrigel pre-coated plates $\left(100 \mu \mathrm{L} / \mathrm{cm}^{2}, 37{ }^{\circ} \mathrm{C}\right.$ for $\left.1 \mathrm{~h}\right)$ and incubated for 7 days in $1 \%$ FBS EMEM/Hams-F12 with $10 \mu \mathrm{M}$ Retinoic Acid (Sigma-Aldrich, Sydney, Australia). The media was replaced every two days. 


\subsection{Transfection of Phosphorodiamidate Morpholino Oligomers and Peptide Conjugated PMOs}

Human and mouse PMOs were purchased from Gene Tools LLC (Philomath, OR, U.S.A.) The human PepK-conjugated PPMO was provided by Sarepta Therapeutics (Cambridge, MA, U.S.A.), and the mouse PepK-conjugated PPMO was synthesised by Cambridge Research Biochemicals (Cleveland, UK) and provided by PYC Therapeutics (Perth, Australia). The PepK chemical composition can be found in [32] and the PPMO structure illustrated in Figure 1c. PMOs and PPMOs were transfected un-complexed at doses ranging from $100 \mathrm{nM}$ to $10 \mu \mathrm{M}$. P/PMOs were diluted accordingly in OptiMEM and applied directly to the cells. Transfected cells were incubated at $37^{\circ} \mathrm{C}$ for 3 days prior to cell harvesting.

\subsection{RNA Extraction, Polymerase Chain Reaction, Visualisation and Analysis}

RNA was extracted using the MagMAX-96 Total RNA Isolation Kit, including a DNase treatment (Life Technologies), according to the manufacturer's instructions. RT-PCRs were performed using the One-step Superscript III RT-PCR kit with Platinum Taq polymerase (Life Technologies) according to the manufacturer's instructions. All primer sequences and PCR conditions used in this study are detailed in Table 2. All PCR products were separated on a $2 \%$ agarose gel, stained with RedSafe (iNtRON Biotechnology, Korea) and visualized on a Vilber Lourmat Fusion FX System (Germany). Bio-1D Software (Vilber Lourmat) was used for densitometry analysis.

Table 2. PCR conditions for human SMN and mouse Smn transcript amplification.

\begin{tabular}{|c|c|c|c|}
\hline Transcript Sizes & Primer & Primer Sequence & Temperature Profile \\
\hline $\begin{array}{c}S M N \\
\text { FL } 404 \mathrm{bp} \\
\Delta 5308 \mathrm{bp} \\
\Delta 7350 \mathrm{bp}\end{array}$ & $\begin{array}{l}\text { Exon } 4 \text { Fwd } \\
\text { Exon } 8 \text { Rev }\end{array}$ & $\begin{array}{c}\text { AGGTCTCCTGGAAATAAATCAG } \\
\text { TGGTGTCATTTAGTGCTGCTCT }\end{array}$ & $\begin{array}{c}55^{\circ} \mathrm{C} 30 \mathrm{~min} \\
94^{\circ} \mathrm{C} 2 \mathrm{~min} \\
25 \mathrm{cycles}: \\
94^{\circ} \mathrm{C} 40 \mathrm{~s} \\
56^{\circ} \mathrm{C} 1 \mathrm{~min} \\
68^{\circ} \mathrm{C} 1 \mathrm{~min}\end{array}$ \\
\hline 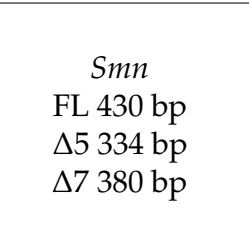 & $\begin{array}{l}\text { Exon } 4 \text { Fwd } \\
\text { Exon } 8 \text { Rev }\end{array}$ & $\begin{array}{l}\text { GAAAGTCAAGTTTCCACAGACG } \\
\text { CACCCCATCTCCTGAGACAGAGC }\end{array}$ & $\begin{array}{c}55^{\circ} \mathrm{C} 30 \mathrm{~min} \\
94^{\circ} \mathrm{C} 2 \mathrm{~min} \\
28 \mathrm{cycles}: \\
94^{\circ} \mathrm{C} 40 \mathrm{~s} \\
60^{\circ} \mathrm{C} 1 \mathrm{~min} \\
68^{\circ} \mathrm{C} 1 \mathrm{~min}\end{array}$ \\
\hline
\end{tabular}

\subsection{In Silico Analysis of SMN and Smn Splice Site Scores}

In silico analysis of the human and mouse exon 5 and 7 splice sites was preformed using the MaxEntScan (MES) prediction tool (available online: http:/ /hollywood.mit.edu/ burgelab/maxent/Xmaxentscan_scoreseq_acc.html, last accessed 19 February 2021) [33] to determine the predictive strength of the respective splice sites, based on the maximum entropy principle.

\subsection{In Vivo Evaluation of a Peptide-Conjugated PMO}

C57BL/10ScSnDmd ${ }^{\mathrm{mdx}}(\mathrm{mdx})$ mice were supplied by the Animal Resources Centre (Murdoch, Australia) and housed at the Small Animal Facility, Murdoch University according to the National Health and Medical Research Council Code of Practice. All experiments performed on animals were approved by the Murdoch University Animal Ethics Committee (approval number R2625/13). As a proof of concept experiment and to determine dosage for future experiments, an $m d x$ pup (postnatal day 5 (P5)) was injected with a single subcutaneous injection $(2 \mathrm{nmol})$ and a single intraperitoneal injection $(2 \mathrm{nmol})$ (combined oligomer dosage of $4 \mathrm{nmol}$ ) of PepK peptide-conjugated PMO in normal saline. The Smn-PMO and the PepK peptide alone were used as delivery controls, whereby $m d x$ mice were treated with 3 subcutaneous injections $(2 \mathrm{nmol}$ ) on P5, P7 and P9 (combined PMO or peptide dosage of $6 \mathrm{nmol}$ ). Treated mice were sacrificed 7 days following the initial 
injection and tissue sampled and cryopreserved for Smn detection. Tissues were sectioned on a cryostat and lysed for RNA extraction and RT-PCR analysis as above.

\section{Results}

\subsection{Screening of 2'-O-Methyl AOs Targeting SMN Exons 5 and 7 in Normal Human Fibroblasts}

Antisense oligomers composed of 2'-O-methyl modified bases on a phosphorothioate backbone were designed to target acceptor and donor splice sites and ESE domains across exons 5 and 7 of the SMN1/SMN2 genes. Initially, AOs designed to induce SMN exon 7 skipping were transfected into normal human fibroblasts using Lipofectamine 3000 (Life Technologies, Melbourne, Australia) at 100, 50 and 25 nM. RT-PCR analysis and gel fractionation (Figure 2) revealed a larger product above the expected full-length amplicon, consistent with a PCR heteroduplex. Antisense oligomers designed to target exon 7 were found be effective at inducing exon 7 skipping, with a clear dose response induced by all AOs tested. Sequences targeting the acceptor splice site and internal ESEs appear to be the most effective, with up to $90 \%$ exon skipping induced by the majority of AOs at $100 \mathrm{nM}$, while an AO targeting the donor splice site was clearly less efficient with only $23 \%$ exon 7 skipping in cells after transfection at the higher concentration. AOs h3 (25mer), $\mathrm{h} 4$ (22mer) and h5 (20mer) all induced the highest levels of exon skipping at $100 \mathrm{nM}$, and therefore, these sequences could be interchangeable as positive controls if AOs of different lengths were needed. However, consistent with the principle of using the shorter AOs when possible, $\mathrm{h} 5$ was selected for future studies. When comparing the sequences reported here with those reported by others [25], there was no notable difference in exon skipping effect (not shown).
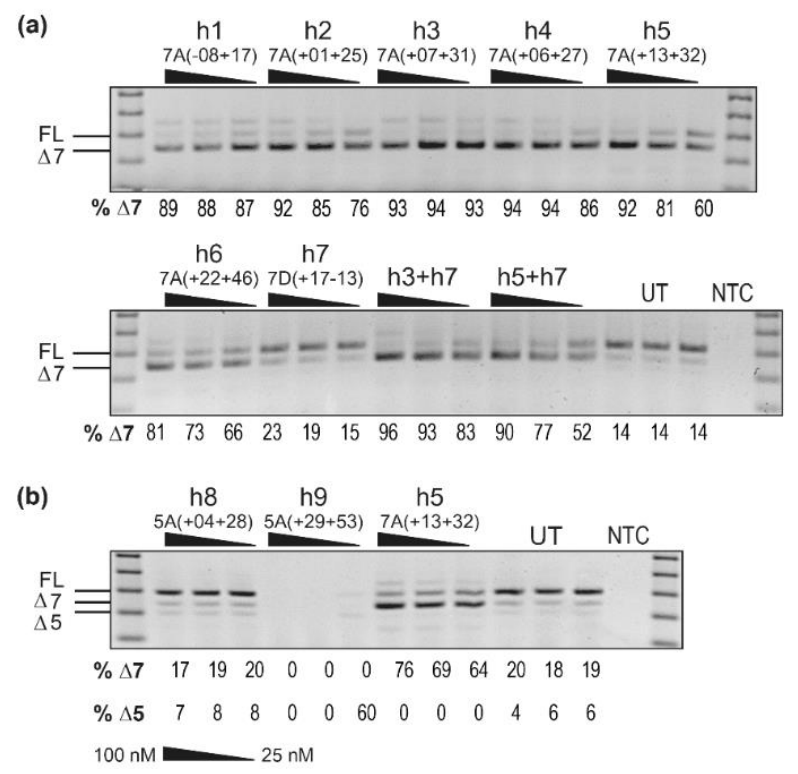

Figure 2. RT-PCR analysis of $S M N$ transcripts across exons 4-8 in normal human fibroblasts transfected with 2'-O-methyl PS AOs (100, 50 and $25 \mathrm{nM}$ ). The full length transcript (FL, $404 \mathrm{bp}$ ) and exon skipped products (delta- $7(\Delta 7), 350 \mathrm{bp}$ and delta-5( $\Delta 5), 308 \mathrm{bp}$ ) are indicated by the lines to the left of the gel, and the percentage of (a) $\triangle 7-S M N$ or (b) $\triangle 7-S M N$ and $\triangle 5-S M N$ as determined by densitometry is shown below each lane for comparison to controls and untreated (UT) fibroblasts. A 100 bp size marker was used for size comparison and a no template RT-PCR control (NTC) was loaded in the final lane.

To ascertain whether exon skipping could be enhanced by non-overlapping AOs in combination, two cocktails were tested, combining the $\mathrm{h} 7$ donor site $\mathrm{AO}$ with effective AOs targeting the middle of the exon, $\mathrm{h} 3(\mathrm{H} 7 \mathrm{~A}(+07+31))$, and $\mathrm{h} 5(\mathrm{H} 7 \mathrm{~A}(+13+32))$ at a $1: 1$ ratio. These cocktails induced lower levels of exon 7 skipping compared to the h3 and h5 AOs alone. 
To compare AO-mediated exon 5 and 7 skipping from the human $S M N$ transcripts, two exon 5 skipping AOs were tested in normal human fibroblasts, for comparison to exon 7 skipping (Figure 2b). Analysis of predicted exon 5 and 7 splice site maximum entropy scores using MaxEntScan [33] (Table 3) revealed exon 5 to be the "weaker" exon with an acceptor site MES score of 5.44, compared to exon 7 with an acceptor score of 10.92. Contrary to this observation, and although removal of exon 5 can still produce a semi-functional transcript [34], AO-induced exon 5 skipping in the human transcript was less efficient than exon 7 skipping. AO h8 (SMN H5A $(+04+28)$ ) was not effective, with only 7\% of transcripts showing skipping of exon 5, while AO h9 targeting SMN H5A +29 + 53) appears to completely knockdown full length $S M N$ transcript levels.

Table 3. Predicted splice site scores of human SMN1 and mouse Smn exons 5 and 7 using MaxEntScan. Nucleotides in CAPITALS indicate exon sequence, lower case nucleotides represent intronic sequences.

\begin{tabular}{|c|c|c|c|c|}
\hline Species & Exon & Splice Site & Splice Site Sequence & MES Score \\
\hline \multirow{4}{*}{ Human } & \multirow{2}{*}{5} & Acceptor & ctttgaaatattccttatag CCA & 5.44 \\
\hline & & Donor & CCAgtaagt & 9.09 \\
\hline & \multirow{2}{*}{7} & Acceptor & ttcctttattttccttacagGGT & 10.92 \\
\hline & & Donor & GGAgtaagt & 8.57 \\
\hline \multirow{4}{*}{ Mouse } & \multirow{2}{*}{5} & Acceptor & cttggaaatattctttatagCCA & 3.29 \\
\hline & & Donor & CCAgtaagt & 9.09 \\
\hline & \multirow{2}{*}{7} & Acceptor & tttatatgctctctttacagGGT & 11.13 \\
\hline & & Donor & GAAgtaagt & 9.82 \\
\hline
\end{tabular}

\subsection{In Silico Analysis of Human and Mouse Exon Splice Site Scores}

In silico analysis of the human and mouse exon 5 and 7 splice sites was preformed using the MaxEntScan (MES) prediction tool (available at http://hollywood.mit.edu/ burgelab /maxent/Xmaxentscan_scoreseq_acc.html, accessed on 19 February 2021) [33] to determine the predictive strength of the splice sites, based on the maximum entropy principle (Table 3). Within both the human and mouse gene transcripts, the exon 5 acceptor site had low predicted MES scores, 5.44 and 3.29, respectively, out of a possible 12, suggesting that this is a weak splice site in both species, particularly so in the mouse. While there was no obvious difference in the MES scores for the exon 7 acceptor sites across the species, the mouse exon 7 donor site was predicted to be slightly stronger (9.82) when compared to the human splice motif (8.57); however, this 13\% difference in MES score did not meet the $15 \%$ threshold predicted to alter splicing outcomes [35].

3.3. Using the SMN Exon Skipping AO as a Positive Control for Optimising AO Chemistry and Delivery Across Cell Lines

Following identification of the lead human SMN exon skipping sequence, the h5 sequence was used as a positive control to optimize and compare $\mathrm{AO}$ chemistries and delivery into different cell types. The $\mathrm{h} 5$ sequence targeting $\mathrm{H} 7 \mathrm{~A}(+13+32)$ was synthesized as a more clinically applicable PMO antisense chemistry (SMN-PMO), due to its excellent safety profile. To enhance PMO delivery, we compared the PMO alone and when conjugated to $\mathrm{PepK}$, an arginine-rich cell penetrating peptide (SMN-PPMO), to enhance cellular uptake and exon skipping effect [32].

The SMN-PMO and PPMO were evaluated in human fibroblasts, transfected without a delivery agent at 10, 5, 2.5, 1 and $0.5 \mu \mathrm{M}$, and the transfected cells incubated for 3 days. RT-PCR analysis and gel fractionation of $S M N$ transcripts (Figure 3a) revealed the PMO to be inefficient when delivered uncomplexed, with a maximum of $38 \%$ of transcripts missing exon 7 after transfection at the higher concentration, compared to $15 \%$ in untreated cells. In comparison, the PepK-conjugated PPMO was particularly effective, with 100\% exon 7 skipping induced at all concentrations tested. The presence of a smaller $254 \mathrm{bp}$ 
PCR product was confirmed to be the $S M N$ transcript missing both exons 5 and 7 , and endogenous $\triangle 5-S M N$ was also detected in untreated samples from these cells.

(a)
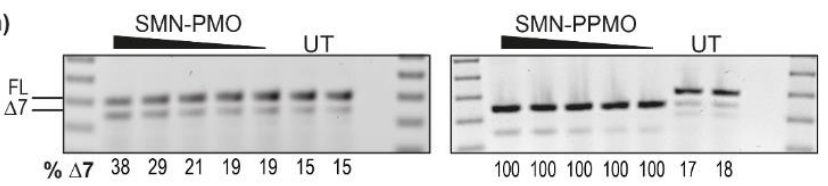

(b)

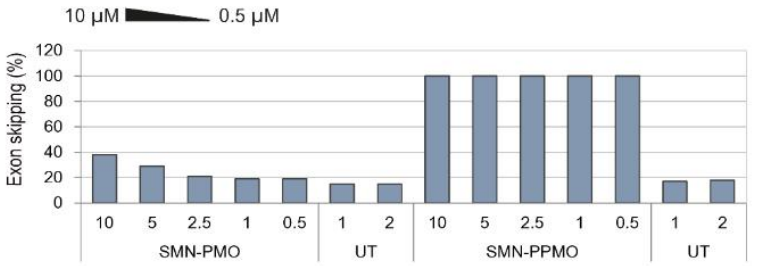

(c) Fibroblasts

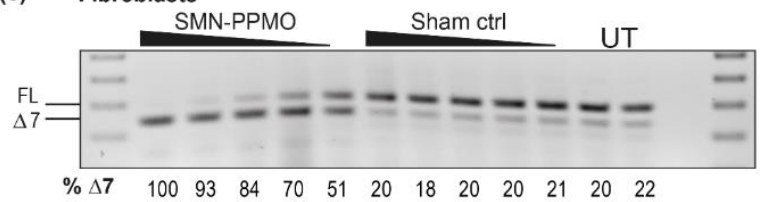

Differentiated SH-SY5Y cells

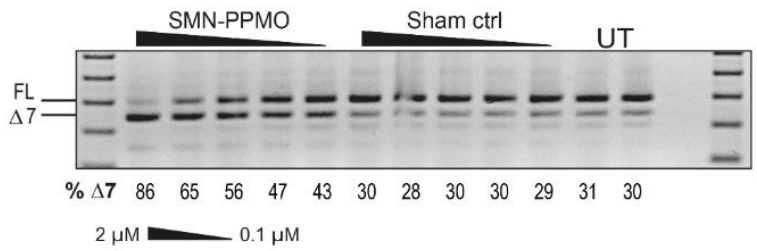

(d)

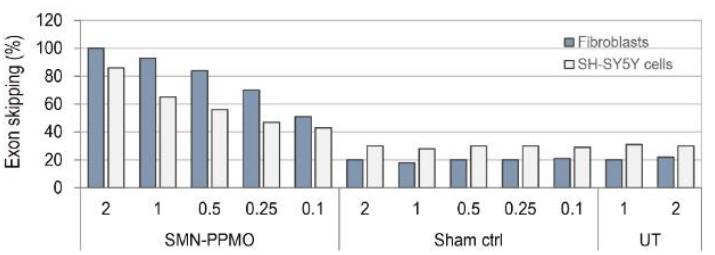

Figure 3. Analysis of AO chemistry and delivery after transfection into fibroblasts and neuronal cells, showing (a) RT-PCR across SMN transcripts following transfection with exon skipping PMO and PPMO into fibroblasts at 10, 5, 2.5, 1 and $0.5 \mu \mathrm{M}$; (b) graphical representation of densitometry as in (a); (c) RT-PCR of SMN transcripts in fibroblasts and SH-SY5Y cells, following transfection with the SMN-PPMO at 2, 1, 0.5, 0.25 and $0.1 \mu \mathrm{M}$; and (d) graphical representation of densitometry as in (c). The full length transcript (FL, $404 \mathrm{bp}$ ) and exon 7 skipped product (delta-7( $\Delta 7), 350 \mathrm{bp}$ ) are indicated by the lines to the left of the gel, and the percentage of $\triangle 7-S M N$ transcript, as determined by densitometry is shown below each lane for comparison to controls and untreated (UT) cells. A 100 bp size marker was used for size comparison and a no template RT-PCR control (NTC) was loaded in the final lane.

The SMN-PPMO was used as a positive control to optimize transfection concentration and PMO delivery into neuronal cells, compared to fibroblasts. For neuronal evaluation, the SH-SY5Y neuroblastoma cell line was differentiated into neurons using $10 \mu \mathrm{M}$ retinoic acid. Fibroblasts and differentiated SH-SY5Y cells were then transfected with SMN-PPMO at 2, 1, 0.5, 0.25 and $0.1 \mu \mathrm{M}$, and incubated for 3 days. RT-PCR analysis of $S M N$ transcripts (Figure 3c) shows $86 \% \triangle 7-S M N$ transcript in cells transfected with the SMN-PPMO at $2 \mu \mathrm{M}$, with a clear dose response. Interestingly, exon 7 skipping was less efficient in the neurons compared to primary fibroblasts, whereby $100 \%$ skipping was observed in cells transfected with the SMN-PPMO at a concentration of $2 \mu \mathrm{M}$. 


\subsection{Screening of 2'-O-Methyl AOs Targeting Smn Exon 7 in Mdx Myoblasts}

To optimize a positive control AO targeting the mouse Smn sequence, 2'-O-methyl AOs targeting the mouse Smn exon 7 were transfected in $m d x$ mouse myoblasts to assess exon skipping. AOs were designed to target the same co-ordinates as the human-specific $\mathrm{AOs}$, and to alternative regions, depending on in silico predictions of splicing factor binding motifs. AOs were transfected into myoblasts using Lipofectamine 2000 (Life Technologies) at 200, 100 and $50 \mathrm{nM}$, with the RT-PCR result of Smn exon 7 skipping shown in Figure 4a. There appears to be a maximum of around $60 \%$ AO-induced exon 7 skipping from the mouse transcript, compared to over $90 \%$ AO-induced skipping from the human SMN transcripts. Unlike the human-specific AOs, targeting the donor splice site of the mouse exon 7 appears to cause the highest levels of skipping, with AOs $\mathrm{m} 6$ and $\mathrm{m} 8$ targeting M7D $(+11-14)$ and M7D $(+17-13)$ respectively, both inducing over $60 \%$ exon 7 skipping. Similar to exon 7 skipping evaluation in human cells, evaluation of AO cocktails, in an attempt to improve exon skipping, did not improve the level of Smn exon skipping (Supplementary Figure S1).

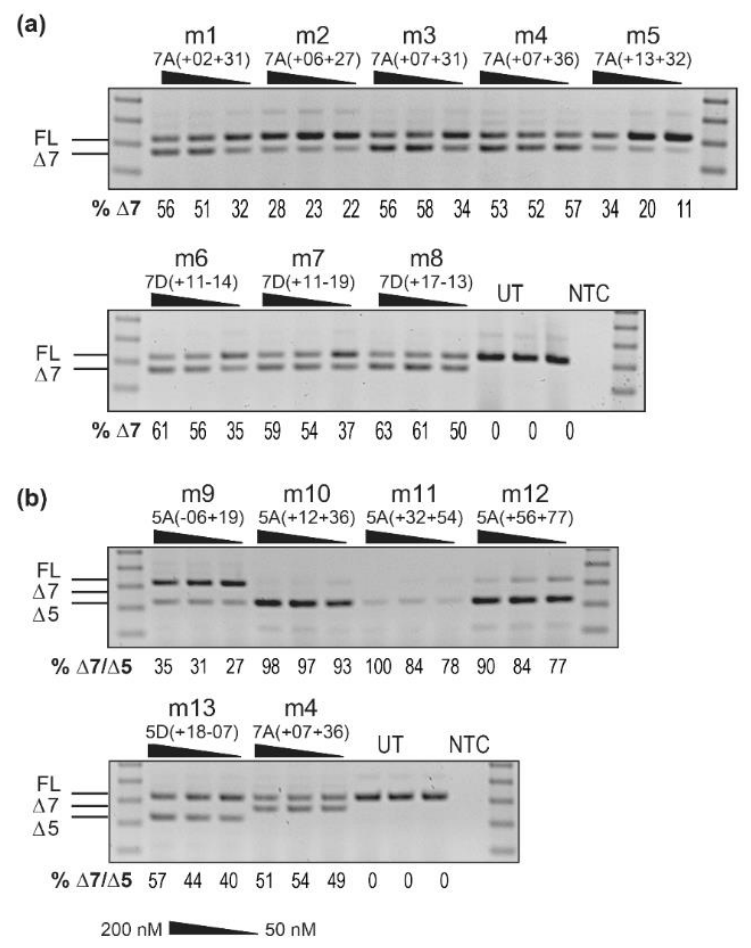

Figure 4. RT-PCR analysis of Smn transcripts across exons 4 to 8 in $m d x$ mouse myoblasts. Myoblasts were transfected with individual 2'-O-methyl PS AOs (200, 100 and $50 \mathrm{nM}$ ), targeting (a) exon 7, and (b) exons 5 and 7 . The full length transcript (FL, $430 \mathrm{bp}$ ) and exon skipped products (delta-7( $\Delta 7)$, $380 \mathrm{bp}$ and delta-5( $\Delta 5), 334 \mathrm{bp}$ ) are indicated by the lines to the left of the gel. The percentage of $\Delta 7-S m n$ and $\Delta 5$-Smn transcripts, as determined by densitometry, are shown below each lane for comparison to controls of untreated (UT) myoblasts. A 100 bp size marker was used for size comparison and a no template RT-PCR control (NTC) was loaded in the final lane.

To compare the skipping of mouse Smn exons 5 and 7, 2'-O-methyl, AOs targeting exon 5 and exon 7 of the mouse sequence were tested at 200, 100 and $50 \mathrm{nM}$. RT-PCR results (Figure $4 \mathrm{~b}$ ) show that $\mathrm{m} 4$, the exon 730 -mer targeting M7A(+ $07+36$ ), induces a maximum of $50 \%$ exon 7 skipping in mouse myoblasts, while AOs targeting Smn exon 5 induce close to $100 \% \Delta 5-\mathrm{S} m n$ transcripts. The sequence $\mathrm{m} 10$, a 25 -mer targeting $\mathrm{M} 5 \mathrm{~A}(+12$ +36), induces greater than $90 \%$ skipping at $50 \mathrm{nM}$, suggesting that AOs targeting exon 5 are more effective as positive controls for mouse studies. 


\subsection{Using the Mouse Smn AO as a Positive Control for Optimizing AO Chemistry and In Vivo Delivery}

The best exon 7 and exon 5 skipping AOs targeting the mouse sequence ( $\mathrm{m} 4$ and $\mathrm{m} 10)$ were synthesized as unmodified PMOs and transfected uncomplexed at 20, 10, 5, 2.5 and $1 \mu \mathrm{M}$ into $m d x$ myoblasts, and the transfected cells incubated for 3 days. Interestingly, RT-PCR results (Figure 5a) show the PMOs to be equally effective at inducing skipping of their target exons, with a maximum of $54-55 \%$ exon skipping achieved in myoblasts transfected with either PMO. Looking at the levels of exon skipping at the low 2.5 and $1 \mu \mathrm{M}$ doses, it appears that the PMO targeting exon 7 may be marginally more effective than the PMO targeting exon 5, inconsistent with the earlier results obtained when testing 2'-O-methyl PS AOs.

(a)

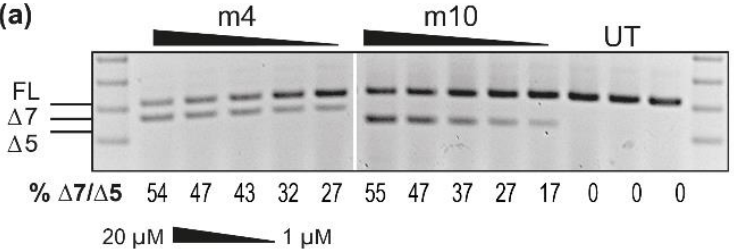

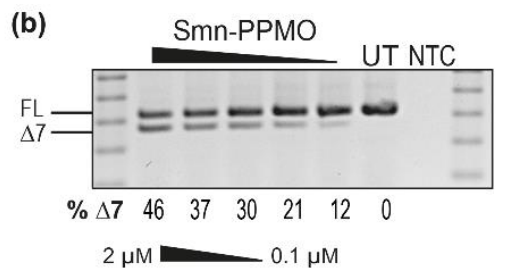

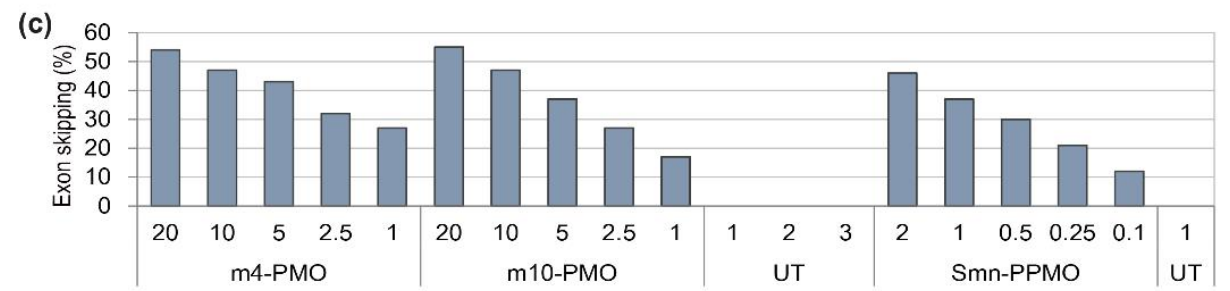

(d)

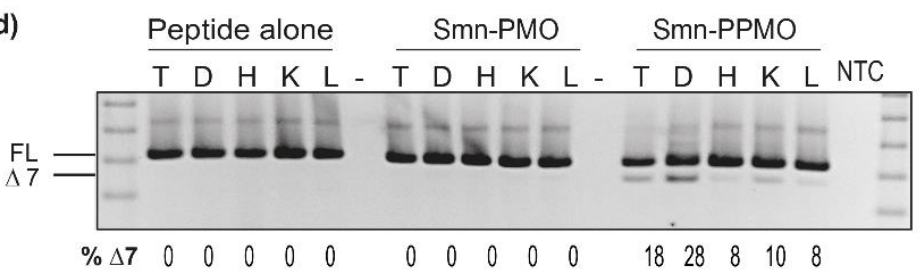

(e)

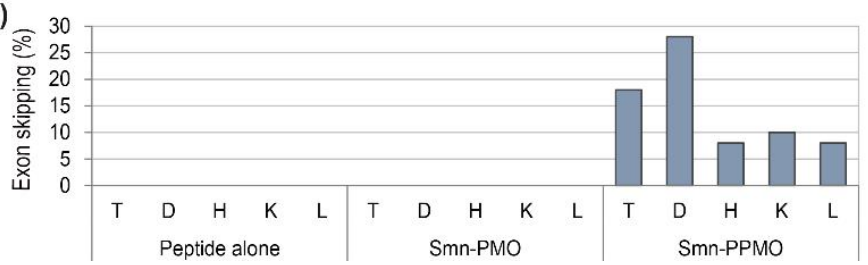

Figure 5. RT-PCR analysis of Smn products across exons 4 to 8 in; (a) $m d x$ mouse myoblasts transfected with the exon 7 and 5 PMOs $(20,10,5,2.5$, and $1 \mu \mathrm{M})$ and compared to untreated (UT) myoblasts; (b) $m d x$ mouse myoblasts transfected the exon 7 Smn-PPMO $(2,1,0.5,0.25$ and $0.1 \mu \mathrm{M})$ and compared to untreated (UT) myoblasts, with the graphed densitometry of (a) and (b) shown in (c) and (d) tissue harvested from $m d x$ mice treated with either an un-conjugated peptide, unconjugated PMO ( $3 x$ subcutaneous ( $2 \mathrm{nmol}$ ) injections) or the Smn-PPMO (1x subcutaneous ( $2 \mathrm{nmol})$ and $1 \mathrm{x}$ intraperitoneal ( $2 \mathrm{nmol})$ injections), with the graphed densitometry shown in (e). Tissues evaluated include the tibialis anterior (T), diaphragm (D), heart $(\mathrm{H})$, kidney $(\mathrm{K})$ and liver $(\mathrm{L})$. The full length transcript (FL, $430 \mathrm{bp}$ ) and exon skipped products (delta-7( $\Delta 7), 380 \mathrm{bp})$ are indicated by the lines to the left of the gel. The percentage of $\Delta 7-S m n$ transcripts, as determined by densitometry, are shown below each lane. A 100 bp size marker was used for size comparison and a no template RT-PCR control (NTC) was loaded in the final lane.

Given that the exon 7 skipping PMO was most effective, and that 25 bases is preferred to reduce the cost and maintain ease of PMO synthesis, the $\mathbf{m} 3$ sequence, targeting m7A $(+07+31)$, was chosen as a positive control to take forward for in vitro evaluation as a PPMO. The Smn-PPMO was transfected into $m d x$ myoblasts $(2,1,0.5,0.25$ and $0.1 \mu \mathrm{M})$, 
and the transfected cells incubated for 3 days prior to Smn analysis (Figure 5b). Although increased levels of exon 7 skipping were observed using these concentrations in human cells, the mouse-specific Smn-PPMO only induced a maximum of $45 \%$ exon 7 skipping in mouse cells. This finding was consistent with the results obtained when testing 2'-O-methyl PS AOs in mouse cells, further supporting the concept that mouse-derived muscle cells may have a threshold of $50 \%$ exon 7 skipping.

To further demonstrate the advantage of a positive control AO sequence, the mousespecific PPMO was evaluated in vivo as proof-of-concept for tissue delivery following chemistry optimization. $M d x$ mouse pups (postnatal day 5) were administered the SmnPPMO by subcutaneous $(2 \mathrm{nmol})$ and intraperitoneal $(2 \mathrm{nmol})$ administration. The peptideonly control and the Smn-PMO were included as controls and administered to $m d x$ mouse pups three times subcutaneously $(2 \mathrm{nmol})$, with each injection administered at different sites (flanks and scruff) over five days. Seven days after the initial treatment, mice were sacrificed and tibialis anterior muscle (T), diaphragm (D), heart (H), kidney (K) and liver (L) were sampled for Smn analysis (Figure 5d). While no Smn exon 7 skipping was detected in mice treated with the uncomplexed PMO or the peptide alone, clear exon 7 skipping was evident in all tissues evaluated from the Smn-PPMO-treated mice, including a hint of exon skipping ( $8 \%$ ) observed in the myocardium, a notoriously difficult-to-transfect tissue $[36,37]$.

\section{Discussion}

The number and diversity of antisense applications has greatly expanded over the past decade, and as a result, so too has the art of AO design and optimization. Refinements and modifications to $\mathrm{AO}$ chemistries and subsequent methods for enhancing delivery continue to evolve. For efficient optimization of AO chemistry and delivery, we suggest that a positive control $\mathrm{AO}$, targeting a ubiquitously expressed gene transcript, should be used during novel $\mathrm{AO}$ evaluation. Here, we report $\mathrm{AO}$ sequences targeting the human and mouse SMN / Smn genes that could be utilized in both in vitro and in vivo models to assess AO delivery.

Antisense oligomers were initially designed to induce skipping of the alternatively spliced exons 5 and 7 from $S M N 1 / S M N 2$ in normal human fibroblasts. Antisense oligomers designed to target across exon 7 were very effective at inducing exon skipping, with over $90 \%$ skipping of the target exon induced in cells transfected with $100 \mathrm{nM}$ of the AO. The most efficient $\mathrm{AO}$ sequences, $\mathrm{H7A}(+07+31)$ and $\mathrm{H7A}(+13+32)$, anneal over an exonic splicing enhancer site, predicted to recruit binding of a number of positive splicing factors, predominately, Tra $2 \beta$. These AOs were both effective at inducing almost complete exon skipping and could be used interchangeably when different $\mathrm{AO}$ sequence lengths are preferred.

Interestingly, while $S M N$ exon 5 had a weaker predicted splice site score when compared to exon 7 (Table 3), efficient exon 5 skipping was not achieved by either AO transfected in human fibroblasts. Of particular note, one exon 5 targeting AO, $\mathrm{H} 5 \mathrm{~A}(+29+53)$, induced up to $100 \%$ transcript knockdown, with a similar result observed by the homologous mouse-specific $\mathrm{AO}$ targeting the same region, $\mathrm{M} 5 \mathrm{~A}(+32+56)$. These AOs target a somewhat repetitive region and have a high guanine $(\mathrm{G})$ content (17 out of 25 bases), and as such have very high melting temperatures of $87^{\circ} \mathrm{C}$ and $86^{\circ} \mathrm{C}$, respectively. High G-content AOs with contiguous stretches of Gs are reported to induce G-quartets and unique secondary structures that can have a multitude of effects on cell biology, including interaction with transcription inhibitors [38]. Taken together, these factors may further exacerbate the reported off-target binding effects of negatively charged phosphorothioate AOs, which we have previously shown to influence SMN transcript levels [14]. Intriguingly, AOs with a higher G-content were observed in this prior study to induce off-target protein accumulation within the cytoplasm. Indeed, these sequences are very G-rich, and are challenging for PMO synthesis with many commercial suppliers reluctant to make these 
compounds. Given that the results of exon 7 skipping were so impressive, we chose not to further pursue AOs to induce exon 5 skipping from the human gene transcript.

As a proof-of-concept for optimizing AO chemistry and delivery into multiple cell lines, the lead human SMN exon 7 skipping $\mathrm{AO}$ sequence was synthesized as a PMO, and a PMO conjugated to a PepK cell penetrating peptide tag to enhance delivery (PPMO) [32]. As expected, PepK dramatically improved cellular uptake and AO-induced SMN splicing efficiency when evaluated in normal human fibroblasts. Antisense oligomer delivery into fibroblasts is well established and routinely performed in our laboratory; however, AO delivery into neuronal cells is less efficient and requires an assisted delivery, such as PepKmediated enhanced cellular uptake. To establish the effective concentration required for delivery into neuronal cells, the SMN-PPMO was evaluated in differentiated SH-SY5Y cells. The SMN-PPMO was highly effective at inducing SMN exon 7 skipping at the higher concentrations, confirming the use of the AO sequence as a positive control for neuronal cells.

To identify a positive control AO for use in mouse cells and for in vivo studies, mouse $\mathrm{S} m n$-specific sequences were designed and transfected into $m d x$ myogenic cells. Interestingly, mouse-specific PMOs were only able to induce up to $45-60 \%$ exon 7 skipping, compared to the $100 \%$ targeted skipping achieved in primary human fibroblasts. Differences in the predictive strength of the exon 7 splice site scores between the species did not meet the threshold expected to alter splicing outcomes and may not fully account for the differences in exon skipping efficiency. Transcripts from both the SMN1 and SMN2 genes are amplified from the human RNA, and, therefore, the endogenous excision of exon 7 that is the result of poorer exon 7 selection in the SMN2 gene transcripts, unique to human cells and not present in mice, could in part explain the difference in exon 7 skipping efficiency between species.

Differences in splicing factors and splicing regulation between species may also contribute to differences in AO-mediated exon-skipping effectiveness. We have previously reported these differences in $\mathrm{AO}$ targeting of the human and mouse dystrophin gene transcripts [26]. Dystrophin transcript exon skipping studies have shown that certain exons are more difficult to skip than others, with some requiring combinations of AOs to achieve efficient exon skipping [17]. Transfection of cocktails of AOs targeting mouse Smn exon 7 marginally improved exon skipping levels when compared to transfection of individual AOs, but did not justify the ongoing use of $\mathrm{AO}$ combinations. It appears that only a maximum of $60 \%$ AO-induced skipping of mouse Smn exon 7 can be achieved, compared to the near 100\% skipping induced in the human SMN transcripts. Regardless of how highly conserved the $S M N$ and $D M D$ transcripts are between humans and mice, taken together, these results emphasize the disparities in splicing between the species, possibly reflecting the context of the exon and the influence of intron structure and therefore, the need for species-specific control AO sequences. This finding is also important for the design of therapeutic AOs, whereby species-specific $\mathrm{AO}$ sequences should be designed to target across the exon and be screened prior to in vivo application.

In comparison to 2'-O-methyl AOs targeting Smn exon 7, 2'-O-methyl, AOs targeting Smn exon 5 induced up to $100 \%$ exon 5 skipping in $m d x$ myoblasts, compared to $50 \%$ exon 7 skipping, suggesting that exon 7 splicing is more tightly regulated than exon 5 in the mouse transcript. This finding was supported by the predicted splice site MES scores, suggesting Smn exon 5 to be a less efficiently spliced exon in the mouse model compared to exon 7. Interestingly, when the sequences were synthesized as PMOs, there was no obvious difference in the levels of skipping of the different target exons. We therefore recommend that AOs targeting Smn exon 5 and 7 could be used interchangeably as positive control AOs. However, there may be chemistry-dependent differences in AO-mediated skipping efficiency; indeed, it is not recommended that AOs with such a high CG content be synthesized as PMOs, as this can lead to poor water solubility and may account for reduced antisense activity of the exon 5 PMO [39]. 
The use of a ubiquitously expressed positive control AO sequence is of great importance when optimizing $\mathrm{AO}$ sequence, chemistry, delivery and tissue targeting for in vivo studies. Here, we provide evidence for the use of the Smn exon 7 skipping AO as an ideal control for assessing tissue delivery following systemic $\mathrm{AO}$ administration of two $\mathrm{AO}$ chemistries: the PMO and the PepK-conjugated PPMO. Consistent with our in vitro data, use of the PepK conjugate markedly improved cellular uptake and antisense effects. While mice treated with the unconjugated Smn-PMO showed no evidence of exon 7 skipping in the tissues evaluated, mice treated with the peptide-conjugated Smn-PPMO clearly demonstrated exon 7 skipping within each of the tissues. The use of the positive control PMO permitted exon 7 skipping to be quantified and assessed delivery to specific tissue types prior to evaluation of therapeutic antisense molecules in future studies. This has huge benefits for the development and evaluation of novel AO chemistries or cell penetrating peptides designed for specific cellular delivery and uptake. Given the ubiquitous expression of Smn, this AO sequence can be utilized to assess tissue delivery of a multitude of $\mathrm{AO}$ chemistries via all routes of administration, including those specifically targeting the central nervous system.

\section{Conclusions}

We report positive control AOs targeting the ubiquitously expressed SMN genes, that can be used as transfection controls and for optimization of both in vitro and in vivo experiments. These sequences could be synthesized using any current or future chemistry suitable for steric-blocking applications. Following $\mathrm{AO}$ sequence screening and evaluation of multiple $\mathrm{AO}$ chemistries, we recommend the following sequences (Table 4) as positive control AOs for both human and mouse transfections and for in vivo evaluation:

Table 4. Sequences and binding co-ordinates of recommended positive control exon skipping AOs designed for the human $S M N$ and mouse Smn transcripts.

\begin{tabular}{ccc}
\hline Species & Coordinates & ${\text { Sequence } \mathbf{5}^{\prime}-\mathbf{3}^{\prime}}^{\prime}$ \\
\hline \multirow{2}{*}{ Human } & SMN H7A $(+07+31)$ & ACC UUC CUU CUU UUU GAU UUU GUC U \\
& SMN H7A $(+13+32)$ & CAC CUU CCU UCU UUU UGA UU \\
\hline \multirow{2}{*}{ Mouse } & Smn M7A $(+07+31)$ & ACU UUC CUU CUU UUU UAU UUU GUC U \\
& Smn M5A $(+12+36)$ & AGG CGG CGG CGG CGG GCC GUU GAA U \\
\hline
\end{tabular}

Supplementary Materials: The following are available online at https://www.mdpi.com/article/10 .3390 / biomedicines 9050552 / s1, Figure S1: Analysis of cocktails of 2'-O-methyl AOs targeting mouse Smn exon 7.

Author Contributions: Conceptualization, L.L.F., S.F., C.M., S.D.W.; experimental design, L.L.F., C.M., A.A., A.S., S.F. and S.D.W.; antisense oligonucleotide design, L.L.F. and C.M.; performed experiments, L.L.F., A.A., I.L.P., and S.F.; performed animal experiments, S.F. and A.A.; writing original draft preparation, L.L.F.; writing, editing and reviewing, S.F. and S.D.W. All authors have read and agreed to the published version of the manuscript.

Funding: This project was funded by the Parry Foundation and Spinal muscular atrophy Association of Australia (2010) and the NHMRC (Project grant 1086311, approved November 2015). L.L.F. and I.L.P. received postgraduate scholarships from Team Spencer and Muscular Dystrophy WA (2012 and 2016).

Institutional Review Board Statement: This study was conducted according to the National Health and Medical Research Council Code of Practice. All experiments performed on animals were approved by the Murdoch University Animal Ethics Committee, Protocol number R2625/13, Approved on 21 February 2014.

Data Availability Statement: Not applicable.

Acknowledgments: The authors would like to acknowledge and thank Sarepta Therapeutics and PYC Therapeutics for providing PPMOs for use in this study. 
Conflicts of Interest: The authors declare no conflict of interest. The funders had no role in the design of the study; in the collection, analyses, or interpretation of data; in the writing of the manuscript, or in the decision to publish the results.

\author{
Abbreviations \\ AO antisense oligonucleotide \\ SMN survival motor neuron \\ SMA spinal muscular atrophy \\ PMO phosphorodiamidate morpholino oligomer \\ PPMO peptide-conjugated phosphorodiamidate morpholino oligomer
}

\title{
References
}

1. Uehara, T.C.J.; Choong, M.; Nakamori, H.; Hayakawa, K.; Nishiyama, Y.; Kasahara, K.; Baba, T.; Nagata, T.; Yokota, H.; Tsuda, S.; et al. Amido-bridged nucleic acid (AmNA)-modified antisense oligonucleotides targeting alpha-synuclein as a novel therapy for Parkinson's disease. Sci. Rep. 2019, 9, 7567. [CrossRef]

2. Nizzardo, M.; Simone, C.; Rizzo, F.; Ulzi, G.; Ramirez, A.; Rizzuti, M.; Bordoni, A.; Bucchia, M.; Gatti, S.; Bresolin, N.; et al. Morpholino-mediated SOD1 reduction ameliorates an amyotrophic lateral sclerosis disease phenotype. Sci. Rep. 2016, 6, 21301. [CrossRef]

3. Aung-Htut, M.T.; Comerford, I.; Johnsen, R.; Foyle, K.; Fletcher, S.; Wilton, S.D. Reduction of integrin alpha 4 activity through splice modulating antisense oligonucleotides. Sci. Rep. 2019, 9, 1-12. [CrossRef]

4. Flynn, L.L.; Mitrpant, C.; Pitout, I.L.; Fletcher, S.; Wilton, S.D. Antisense Oligonucleotide-Mediated Terminal Intron Retention of the SMN2 Transcript. Mol. Ther. Nucleic Acids 2018, 11, 91-102. [CrossRef] [PubMed]

5. Tabaglio, T.; Low, D.H.; Teo, W.K.L.; Goy, P.A.; Cywoniuk, P.; Wollmann, H.; Ho, J.; Tan, D.; Aw, J.; Pavesi, A.; et al. MBNL1 alternative splicing isoforms play opposing roles in cancer. Life Sci. Alliance 2018, 1, e201800157. [CrossRef] [PubMed]

6. Wilton, S.D.; Fall, A.M.; Harding, P.L.; McClorey, G.; Coleman, C.; Fletcher, S. Antisense Oligonucleotide-induced Exon Skipping Across the Human Dystrophin Gene Transcript. Mol. Ther. 2007, 15, 1288-1296. [CrossRef] [PubMed]

7. Pitout, I.; Flynn, L.L.; Wilton, S.D.; Fletcher, S. Antisense-mediated splice intervention to treat human disease: The odyssey continues. F1000Research 2019, 8, 710. [CrossRef]

8. $\quad$ Aung-Htut, M.T.; McIntosh, C.S.; West, K.A.; Fletcher, S.; Wilton, S.D.; Ham, K. In Vitro Validation of Phosphorodiamidate Morpholino Oligomers. Molecules 2019, 24, 2922. [CrossRef]

9. Aung-Htut, M.T.; McIntosh, C.S.; Ham, K.A.; Pitout, I.L.; Flynn, L.L.; Greer, K.; Fletcher, S.; Wilton, S.D. Systematic Approach to Developing Splice Modulating Antisense Oligonucleotides. Int. J. Mol. Sci. 2019, 20, 5030. [CrossRef]

10. Errington, S.J.; Mann, C.J.; Fletcher, S.; Wilton, S.D. Target selection for antisense oligonucleotide induced exon skipping in the dystrophin gene. J. Gene Med. 2003, 5, 518-527. [CrossRef]

11. Wang, T.; Larcher, L.M.; Ma, L.; Veedu, R.N. Systematic Screening of Commonly Used Commercial Transfection Reagents towards Efficient Transfection of Single-Stranded Oligonucleotides. Molecules 2018, 23, 2564. [CrossRef]

12. Linnane, E.; Davey, P.; Zhang, P.; Puri, S.; Edbrooke, M.; Chiarparin, E.; Revenko, A.S.; MacLeod, A.R.; Norman, J.C.; Ross, S.J. Differential uptake, kinetics and mechanisms of intracellular trafficking of next-generation antisense oligonucleotides across human cancer cell lines. Nucleic Acids Res. 2019, 47, 4375-4392. [CrossRef]

13. Wang, S.; Sun, H.; Tanowitz, M.; Liang, X.-H.; Crooke, S.T. Intra-endosomal trafficking mediated by lysobisphosphatidic acid contributes to intracellular release of phosphorothioate-modified antisense oligonucleotides. Nucleic Acids Res. 2017, 45, 5309-5322 [CrossRef]

14. Flynn, L.L.; Ruohan, L.; Aung-Htut, M.T.; Pitout, I.L.; Cooper, J.; Hubbard, A.; Griffiths, L. Interaction of modified oligonucleotides with nuclear proteins, formation of novel nuclear structures and sequence-independent effects on RNA processing. bioRxiv 2019. [CrossRef]

15. Shen, W.; De Hoyos, C.L.; Sun, H.; Vickers, T.A.; Liang, X.-H.; Crooke, S.T. Acute hepatotoxicity of 2' fluoro-modified 5-10-5 gapmer phosphorothioate oligonucleotides in mice correlates with intracellular protein binding and the loss of DBHS proteins. Nucleic Acids Res. 2018, 46, 2204-2217. [CrossRef]

16. Crooke, S.T.; Baker, B.F.; Witztum, J.L.; Kwoh, T.J.; Pham, N.C.; Salgado, N.; McEvoy, B.W.; Cheng, W.; Hughes, S.G.; Bhanot, S.; et al. The Effects of 2'-O-Methoxyethyl Containing Antisense Oligonucleotides on Platelets in Human Clinical Trials. Nucleic Acid Ther. 2017, 27, 121-129. [CrossRef]

17. Adams, A.M.; Harding, P.L.; Iversen, P.L.; Coleman, C.; Fletcher, S.; Wilton, S.D. Antisense oligonucleotide induced exon skipping and the dystrophin gene transcript: Cocktails and chemistries. BMC Mol. Biol. 2007, 8, 57-58. [CrossRef]

18. Tsoumpra, M.K.; Fukumoto, S.; Matsumoto, T.; Takeda, S.; Wood, M.J.; Aoki, Y. Peptide-conjugate antisense based splice-correction for Duchenne muscular dystrophy and other neuromuscular diseases. EBioMedicine 2019, 45, 630-645. [CrossRef]

19. Hoffmann, K.; Milech, N.; Juraja, S.M.; Cunningham, P.T.; Stone, S.R.; Francis, R.W.; Anastasas, M.; Hall, C.M.; Heinrich, T.; Bogdawa, H.M.; et al. A platform for discovery of functional cell-penetrating peptides for efficient multi-cargo intracellular delivery. Sci. Rep. 2018, 8, 1-16. [CrossRef] [PubMed] 
20. Hammond, S.M.; Hazell, G.; Shabanpoor, F.; Saleh, A.F.; Bowerman, M.; Sleigh, J.N.; Meijboom, K.E.; Zhou, H.; Muntoni, F.; Talbot, K.; et al. Systemic peptide-mediated oligonucleotide therapy improves long-term survival in spinal muscular atrophy. Proc. Natl. Acad. Sci. USA 2016, 113, 10962-10967. [CrossRef]

21. Guidotti, G.; Brambilla, L.; Rossi, D. Cell-Penetrating Peptides: From Basic Research to Clinics. Trends Pharmacol. Sci. 2017, 38, 406-424. [CrossRef]

22. Singh, N.K.; Singh, N.N.; Androphy, E.J.; Singh, R.N. Splicing of a Critical Exon of Human Survival Motor Neuron Is Regulated by a Unique Silencer Element Located in the Last Intron. Mol. Cell. Biol. 2006, 26, 1333-1346. [CrossRef] [PubMed]

23. Osman, E.Y.; Miller, M.R.; Robbins, K.L.; Lombardi, A.M.; Atkinson, A.K.; Brehm, A.J.; Lorson, C.L. Morpholino antisense oligonucleotides targeting intronic repressor Element1 improve phenotype in SMA mouse models. Hum. Mol. Genet. 2014, 23, 4832-4845. [CrossRef] [PubMed]

24. Finkel, R.S.; Chiriboga, C.A.; Vajsar, J.; Day, J.W.; Montes, J.; De Vivo, D.C.; Yamashita, M.; Rigo, F.; Hung, G.; Schneider, E.; et al. Treatment of infantile-onset spinal muscular atrophy with nusinersen: A phase 2, open-label, dose-escalation study. Lancet 2016, 388, 3017-3026. [CrossRef]

25. Sahashi, K.; Hua, Y.; Ling, K.K.; Hung, G.; Rigo, F.; Horev, G.; Katsuno, M.; Sobue, G.; Ko, C.-P.; Bennett, C.F.; et al. TSUNAMI: An antisense method to phenocopy splicing-associated diseases in animals. Genes Dev. 2012, 26, 1874-1884. [CrossRef]

26. Mitrpant, C.; Adams, A.M.; Meloni, P.L.; Muntoni, F.; Fletcher, S.; Wilton, S.D. Rational Design of Antisense Oligomers to Induce Dystrophin Exon Skipping. Mol. Ther. 2009, 17, 1418-1426. [CrossRef]

27. Bramsen, J.B.; Ekjems, J. Development of Therapeutic-Grade Small Interfering RNAs by Chemical Engineering. Front. Genet. 2012, 3, 154. [CrossRef]

28. Warren, T.K.; Shurtleff, A.C.; Bavari, S. Advanced morpholino oligomers: A novel approach to antiviral therapy. Antivir. Res. 2012, 94, 80-88. [CrossRef]

29. Piva, F.; Giulietti, M.; Burini, A.B.; Principato, G. SpliceAid 2: A database of human splicing factors expression data and RNA target motifs. Hum. Mutat. 2011, 33, 81-85. [CrossRef] [PubMed]

30. Yeo, E.; Hoon, S.; Venkatesh, B.; Burge, C.B. Variation in sequence and organization of splicing regulatory elements in vertebrate genes. Proc. Natl. Acad. Sci. USA 2004, 101, 15700-15705. [CrossRef] [PubMed]

31. Mann, C.J.; Honeyman, K.; McClorey, G.; Fletcher, S.; Wilton, S.D. Improved antisense oligonucleotide induced exon skipping in themdx mouse model of muscular dystrophy. J. Gene Med. 2002, 4, 644-654. [CrossRef]

32. Jearawiriyapaisarn, N.; Moulton, H.M.; Buckley, B.; Roberts, J.; Sazani, P.; Fucharoen, S.; Iversen, P.L.; Kole, R. Sustained Dystrophin Expression Induced by Peptide-conjugated Morpholino Oligomers in the Muscles of mdx Mice. Mol. Ther. 2008, 16, 1624-1629. [CrossRef] [PubMed]

33. Yeo, G.; Burge, C.B. Maximum entropy modeling of short sequence motifs with applications to RNA splicing signals. In Proceedings of the Seventh Annual International Conference on Computational Molecular Biology RECOMB '03, Berlin, Germany, 10-13 April 2003; Volume 11, pp. 322-331. [CrossRef]

34. Mohaghegh, P.; Rodrigues, N.R.; Owen, N.; Ponting, C.P.; Le, T.T.; Burghes, A.H.; Davies, K.E. Analysis of mutations in the tudor domain of the survival motor neuron protein SMN. Eur. J. Hum. Genet. 1999, 7, 519-525. [CrossRef] [PubMed]

35. Moles-Fernández, A.; Duran-Lozano, L.; Montalban, G.; Bonache, S.; López-Perolio, I.; Menéndez, M.; Santamariña, M.; Behar, R.; Blanco, A.; Carrasco, E.; et al. Computational Tools for Splicing Defect Prediction in Breast/Ovarian Cancer Genes: How Efficient Are They at Predicting RNA Alterations? Front. Genet. 2018, 9, 366. [CrossRef] [PubMed]

36. Betts, C.; Saleh, A.F.; Arzumanov, A.A.; Hammond, S.M.; Godfrey, C.; Coursindel, T.; Gait, M.J.; Wood, M.J. Pip6-PMO, A New Generation of Peptide-oligonucleotide Conjugates With Improved Cardiac Exon Skipping Activity for DMD Treatment. Mol. Ther. Nucleic Acids 2012, 1, e38. [CrossRef] [PubMed]

37. Boisguérin, P.; Deshayes, S.; Gait, M.J.; O’Donovan, L.; Godfrey, C.; Betts, C.A.; Wood, M.J.; Lebleu, B. Delivery of therapeutic oligonucleotides with cell penetrating peptides. Adv. Drug Deliv. Rev. 2015, 87, 52-67. [CrossRef]

38. Jing, N.; Li, Y.; Xiong, W.; Sha, W.; Jing, L.; Tweardy, D.J. G-Quartet Oligonucleotides. Cancer Res. 2004, 64, 6603-6609. [CrossRef]

39. Moulton, J.D.; Yan, Y. Using Morpholinos to Control Gene Expression. Curr. Protoc. Mol. Biol. 2008, 83, 26.8.1-26.8.29. [CrossRef] 